\title{
MIXED QUASI-ÉTALE QUOTIENTS WITH ARBITRARY SINGULARITIES
}

\author{
DAVIDE FRAPPORTI \\ University of Bayreuth, Lehrstuhl Mathematik VIII, \\ Universitaetsstrasse 30, D-95447 Bayreuth, Germany \\ e-mail: Davide.Frapporti@uni-bayreuth.de \\ and ROBERTO PIGNATELLI \\ Dipartimento di Matematica, Università di Trento, \\ Via Sommarive 14, I-38123 Trento, Italy \\ e-mail: Roberto.Pignatelli@unitn.it
}

(Received 5 April 2013; accepted 4 November 2013; first published online 26 August 2014)

\begin{abstract}
A mixed quasi-étale quotient is the quotient of the product of a curve of genus at least 2 with itself by the action of a group which exchanges the two factors and acts freely outside a finite subset. A mixed quasi-étale surface is the minimal resolution of its singularities. We produce an algorithm computing all mixed quasi-étale surfaces with given geometric genus, irregularity and self-intersection of the canonical class. We prove that all irregular mixed quasi-étale surfaces of general type are minimal. As an application, we classify all irregular mixed quasi-étale surfaces of general type with genus equal to the irregularity, and all the regular ones with $K^{2}>0$, thus constructing new examples of surfaces of general type with $\chi=1$. We mention the first example of a minimal surface of general type with $p_{g}=q=1$ and Albanese fibre of genus bigger than $K^{2}$.
\end{abstract}

2000 Mathematics Subject Classification. 14J29, 58E40, 14Q10

Introduction In the last decade, after the seminal paper [16], there has been a growing interest in those surfaces birational to the quotient of the product of two curves of genus at least 2 by the action of a subgroup of its automorphism group.

These have shown to be a very productive source of examples, especially in the very interesting and still mysterious case of the surfaces of general type with $\chi(S)=1$ (equivalently $p_{g}(S)=q(S)$ ). Here and in the following we use the standard notation of the theory of complex surfaces, as in $[3,11]$. For motivation and for the state of the art (few years ago) of the research on the surfaces of general type with $p_{g}=q=0$ we suggest to the reader the survey [8], while some information on the more general case of $\chi(S)=1$ can be found in [7, Section 2]. We just mention here that the case $p_{g}=q \geq 3$ has been classified $[\mathbf{1 2}, \mathbf{2 1}, \mathbf{2 5}, \mathbf{3 1}]$, whereas the case $p_{g}=q \leq 2$ is still rather unknown.

Recently, several new surfaces of general type with $p_{g}=q$ have been constructed as the quotient of a product of two curves by the action of a finite group; see [4-6, 9, 10] for $p_{g}=0,[15,28,32,33]$ for $p_{g}=1$ and $[29,41]$ for $p_{g}=2$. In all these cited papers the authors assume that the action is free, or unmixed, which means that the action is diagonal, induced by actions on the factors. 
Table 1. Mixed q.e. surfaces of general type with $K^{2}>0$ and $p_{g}=q=0$

\begin{tabular}{lcccccc}
\hline$K_{S}^{2}$ & $\mathcal{B}(X)$ & Sign. & $G^{0}$ & $G$ & $H_{1}(S, \mathbb{Z})$ & $\pi_{1}(S)$ \\
\hline 1 & $2 C_{2,1}, 2 D_{2,1}$ & $2^{3}, 4$ & $D_{4} \times \mathbb{Z}_{2}$ & $\mathbb{Z}_{2}^{3} \rtimes \mathbb{Z}_{4}$ & $\mathbb{Z}_{4}$ & $\mathbb{Z}_{4}$ \\
2 & $6 C_{2,1}$ & $2^{5}$ & $\mathbb{Z}_{2}^{3}$ & $\mathbb{Z}_{2}^{2} \rtimes \mathbb{Z}_{4}$ & $\mathbb{Z}_{2} \times \mathbb{Z}_{4}$ & $\mathbb{Z}_{2} \times \mathbb{Z}_{4}$ \\
2 & $6 C_{2,1}$ & $4^{3}$ & $\left(\mathbb{Z}_{2} \times \mathbb{Z}_{4}\right) \rtimes \mathbb{Z}_{4}$ & $\mathrm{G}(64,82)$ & $\mathbb{Z}_{2}^{3}$ & $\mathbb{Z}_{2}^{3}$ \\
2 & $C_{2,1}, 2 D_{2,1}$ & $2^{3}, 4$ & $\mathbb{Z}_{2}^{4} \rtimes \mathbb{Z}_{2}$ & $\mathbb{Z}_{2}^{4} \rtimes \mathbb{Z}_{4}$ & $\mathbb{Z}_{4}$ & $\mathbb{Z}_{4}$ \\
2 & $C_{2,1}, 2 D_{2,1}$ & $2^{2}, 3^{2}$ & $\mathbb{Z}_{3}^{2} \rtimes \mathbb{Z}_{2}$ & $\mathbb{Z}_{3}^{2} \rtimes \mathbb{Z}_{4}$ & $\mathbb{Z}_{3}$ & $\mathbb{Z}_{3}$ \\
2 & $2 C_{4,1}, 3 C_{2,1}$ & $2^{3}, 4$ & $\mathrm{G}(64,73)$ & $\mathrm{G}(128,1535)$ & $\mathbb{Z}_{2}^{3}$ & $\mathbb{Z}_{2}^{3}$ \\
2 & $2 C_{3,1}, 2 C_{3,2}$ & $3^{2}, 4$ & $\mathrm{G}(384,4)$ & $\mathrm{G}(768,1083540)$ & $\mathbb{Z}_{4}$ & $\mathbb{Z}_{4}$ \\
2 & $2 C_{3,1}, 2 C_{3,2}$ & $3^{2}, 4$ & $\mathrm{G}(384,4)$ & $\mathrm{G}(768,1083541)$ & $\mathbb{Z}_{2}^{2}$ & $\mathbb{Z}_{2}^{2}$ \\
3 & $C_{8,3}, C_{8,5}$ & $2^{3}, 8$ & $\mathrm{G}(32,39)$ & $\mathrm{G}(64,42)$ & $\mathbb{Z}_{2} \times \mathbb{Z}_{4}$ & $\mathbb{Z}_{2} \times \mathbb{Z}_{4}$ \\
4 & $4 C_{2,1}$ & $2^{5}$ & $D_{4} \times \mathbb{Z}_{2}$ & $D_{2,8,5} \rtimes \mathbb{Z}_{2}$ & $\mathbb{Z}_{2} \times \mathbb{Z}_{8}$ & $\mathbb{Z}_{2}^{2} \rtimes \mathbb{Z}_{8}$ \\
4 & $4 C_{2,1}$ & $2^{5}$ & $\mathbb{Z}_{2}^{4}$ & $\left(\mathbb{Z}_{2}^{2} \rtimes \mathbb{Z}_{4}\right) \times \mathbb{Z}_{2}$ & $\mathbb{Z}_{2}^{3} \times \mathbb{Z}_{4}$ & $\infty$ \\
4 & $4 C_{2,1}$ & $4^{3}$ & $\mathrm{G}(64,23)$ & $\mathrm{G}(128,836)$ & $\mathbb{Z}_{2}^{3}$ & $\mathbb{Z}_{4}^{2} \rtimes \mathbb{Z}_{2}$ \\
8 & $\emptyset$ & $2^{5}$ & $D_{4} \times \mathbb{Z}_{2}^{2}$ & $\left(D_{2,8,5} \rtimes \mathbb{Z}_{2}\right) \times \mathbb{Z}_{2}$ & $\mathbb{Z}_{2}^{3} \times \mathbb{Z}_{8}$ & $\infty$ \\
8 & $\emptyset$ & $4^{3}$ & $\mathrm{G}(128,36)$ & $\mathrm{G}(256,3,678)$ & $\mathbb{Z}_{4}^{3}$ & $\infty$ \\
8 & $\emptyset$ & $4^{3}$ & $\mathrm{G}(128,36)$ & $\mathrm{G}(256,3,678)$ & $\mathbb{Z}_{2}^{4} \times \mathbb{Z}_{4}$ & $\infty$ \\
8 & $\emptyset$ & $4^{3}$ & $\mathrm{G}(128,36)$ & $\mathrm{G}(256,3,678)$ & $\mathbb{Z}_{2}^{2} \times \mathbb{Z}_{4}^{2}$ & $\infty$ \\
8 & $\emptyset$ & $4^{3}$ & $\mathrm{G}(128,36)$ & $\mathrm{G}(256,3,679)$ & $\mathbb{Z}_{2}^{2} \times \mathbb{Z}_{4}^{2}$ & $\infty$ \\
\hline
\end{tabular}

In [24], the first author considered a more general case, assuming the action to be free outside a finite set of points: it is not difficult to show that this includes both the above cases. We call this case quasi-étale since the induced map into the quotient is quasi-étale in the sense of [17]. Since the above mentioned papers give a satisfactory description of the unmixed case, [24] concentrated on the mixed case, which is the complementary case. After some preliminary results, [24] restricted to the case of surfaces of general type with $p_{g}=0$, and imposed a strong condition on the singularities of the quotient surface, obtaining several interesting new examples.

In this paper, we drop any assumption on the value of $p_{g}$ and the type of singularities.

The situation is as follows: Let $C$ be the Riemann surface of genus $g(C) \geq 2$, and let $G$ be a finite group that acts on $C \times C$. We say that $X=(C \times C) / G$ is a quasi-étale quotient if the action of $G$ is free outside a finite set of points. Let $S \rightarrow X$ be the minimal resolution of the singularities of $X$, we call $S$ a quasi-étale surface. The action is mixed if $G \subset \operatorname{Aut}(C \times C) \cong \operatorname{Aut}(C)^{2} \rtimes \mathbb{Z}_{2}$ is not contained in $\operatorname{Aut}(C)^{2}$; if the action is mixed, we say that $X$ is a mixed q.e. quotient, $S$ is a mixed q.e. surface and we denote by $G^{0} \triangleleft G$ the subgroup $G \cap \operatorname{Aut}(C)^{2}$.

The main result of this paper is an algorithm, which, given three fixed integers $p_{g}, q$ and $K^{2}$, produces all mixed q.e. surfaces with those invariants. We implemented the algorithm in the program MAGMA [26]; the script is available at http: / / www. science. unitn. it/ pignatel/papers/Mixed.magma

As an application, running the program for all possible positive values of $K^{2}$ and $p_{g}=q$, we obtained Theorems A, B and C. Note that the program also works for arbitrary values of $K^{2}, p_{g}$ and $q$, so more surfaces may be produced with it.

THEOREM A. The mixed q.e. surfaces $S$ with $p_{g}=q=0$ and $K^{2}>0$ form the 17 irreducible families collected in Table 1. In all cases $S$ is minimal and of general type.

In Table 1, every row corresponds to an irreducible family. Two columns need some explanation: Column $\mathcal{B}(X)$ represents the basket of singularities of $X$ (see Definition 
2.15), column Sign. gives the signature of the generating vector of $G^{0}$ (see Definition $1.5)$ in a compact way, e.g. $2^{3}, 4$ stands for $(q ; 2,2,2,4)$. Throughout the paper we denote by $\mathbb{Z}_{n}$ the cyclic group of order $n$, by $\mathfrak{S}_{n}$ the symmetric group on $n$ letters, by $A_{n}$ the alternating group on $n$ letters, by $Q_{8}$ the group of quaternions, by $D_{n}$ the dihedral group of order $2 n$, by $D_{p, q, r}$ the group $\left\langle x, y \mid x^{p}=y^{q}=1, x y x^{-1}=y^{r}\right\rangle$, by $B D_{n}$ the group $\left\langle x, y \mid y^{2 n}=x^{2} y^{n}=1, x y x^{-1}=y^{-1}\right\rangle$ and by $\mathrm{G}(\mathrm{a}, \mathrm{b})$ the $b^{\text {th }}$ group of order $a$ in the MAGMA database of finite group.

Note that the 13 rows of this list where the mixed q.e. quotient has only Rational Double Points as singularities were already given in [24], so only four of these surfaces are new: three with $K^{2}=2$ and one with $K^{2}=3$. Moreover, note that some of these surfaces have the same invariants of some of the surfaces in [9], including the fundamental group (see, e.g. the case $K^{2}=4$ ). We do not know if two such surfaces are deformation equivalent or not: it would be interesting to study their moduli spaces.

There may exist more mixed q.e. surfaces of general type with $p_{g}=q=0$ : they would have $K^{2} \leq 0$ and therefore they would not be minimal. The strategy (and the program) works in principle for every value of $K^{2}$. Unfortunately, if it seems to work in the case of $K^{2}=0$ (we have not completed the computations), the case when $K^{2}$ is negative seems to be too hard for our program: indeed we tried this on the best computers at our disposal, but it ran out of memory very quickly.

In the irregular $(q>0)$ case, the situation is, from this point of view, much more clear since we could prove the following theorem.

THEOREM. Let $S$ be an irregular mixed q.e. surface of general type, then $S$ is minimal.

The result does not extend to the unmixed case, counterexamples can be found in [28]. Then we could give a complete classification of the mixed q.e. irregular surfaces of general type with $p_{g}=q$.

THEOREM B. The mixed q.e. surfaces of general type $S$ with $p_{g}=q=1$ form the 19 irreducible families collected in Table 2.

In Table 2, we use the same notation of Table 1; we also report the genus $g_{\text {alb }}$ of a general fibre of the Albanese map and we do not report $\pi_{1}(S)$, which is always infinite. Note that there is a surface with $K_{S}^{2}=6$ and $g_{\text {alg }}=7$; to the best of our knowledge, this is the first example of a minimal surface of general type with $p_{g}=q=1$ and $g_{a l b}>K_{S}^{2}$; we recall that this is not possible for $K_{S}^{2} \leq 3$ by the classification [18-20, 22]. We also note the first example with $K^{2}=6$ and $g_{a l b}=5$. Also, other examples with $4 \leq K^{2} \leq 6$ may be, to the best of our knowledge, new, although other surfaces with those invariants have been already constructed (see [28, 30, 33, 36-38]).

THEOREM C. There exists a unique irreducible family of mixed q.e. surfaces of general type with $p_{g}=q \geq 2$, and it has $p_{g}=2$ and $K^{2}=8$, see Table 3 .

The mixed q.e. surfaces with $K_{S}^{2}=8 \chi(S)$ are those for which the action is free; indeed all the examples in Tables 1,2 and 3 appeared in the papers cited at the beginning of this Introduction. In particular, the list in [29] is the complete list of all q.e. surfaces with $p_{g}=q=2$.

An expanded version of these tables can be downloaded from: http: //www. science. unitn. it/ pignatel/papers/TablesMixed. pdf

The paper is organised as follows. 
Table 2. Mixed q.e. surfaces of general type with $p_{g}=q=1$

\begin{tabular}{lcccccc}
\hline$K_{S}^{2}$ & $g_{\text {alb }}$ & $\mathcal{B}(X)$ & Sign. & $G^{0}$ & $G$ & $H_{1}(S, \mathbb{Z})$ \\
\hline 2 & 2 & $C_{2,1}, 2 D_{2,1}$ & $2^{2}$ & $\mathbb{Z}_{2}$ & $\mathbb{Z}_{4}$ & $\mathbb{Z}^{2}$ \\
2 & 2 & $C_{2,1}, 2 D_{2,1}$ & 2 & $D_{8}$ & $D_{2,8,3}$ & $\mathbb{Z}^{2}$ \\
2 & 2 & $C_{2,1}, 2 D_{2,1}$ & 2 & $Q_{8}$ & $B D_{4}$ & $\mathbb{Z}^{2}$ \\
4 & 3 & $4 C_{2,1}$ & $2^{2}$ & $\mathbb{Z}_{4}$ & $\mathbb{Z}_{8}$ & $\mathbb{Z}_{2} \times \mathbb{Z}^{2}$ \\
4 & 3 & $4 C_{2,1}$ & $2^{2}$ & $\mathbb{Z}_{2} \times \mathbb{Z}_{2}$ & $\mathbb{Z}_{2} \times \mathbb{Z}_{4}$ & $\mathbb{Z}_{2} \times \mathbb{Z}^{2}$ \\
4 & 2 & $4 C_{2,1}$ & 2 & $\mathbb{Z}_{2}^{2} \rtimes \mathbb{Z}_{4}$ & $\mathrm{G}(32,29)$ & $\mathbb{Z}_{2}^{2} \times \mathbb{Z}^{2}$ \\
4 & 3 & $4 C_{2,1}$ & 2 & $D_{4,4,3}$ & $D_{4,8,3}$ & $\mathbb{Z}_{2} \times \mathbb{Z}^{2}$ \\
4 & 3 & $4 C_{2,1}$ & 2 & $D_{4,4,3}$ & $D_{4,8,7}$ & $\mathbb{Z}_{2} \times \mathbb{Z}^{2}$ \\
4 & 2 & $4 C_{2,1}$ & 2 & $D_{4,4,3}$ & $\mathrm{G}(32,32)$ & $\mathbb{Z}_{2}^{2} \times \mathbb{Z}^{2}$ \\
4 & 2 & $4 C_{2,1}$ & 2 & $D_{4,4,3}$ & $\mathrm{G}(32,35)$ & $\mathbb{Z}_{2}^{2} \times \mathbb{Z}^{2}$ \\
4 & 3 & $4 C_{2,1}$ & 2 & $D_{2,8,5}$ & $\mathrm{G}(32,15)$ & $\mathbb{Z}_{2} \times \mathbb{Z}^{2}$ \\
5 & 3 & $C_{3,1}, C_{3,2}$ & 3 & $B D_{3}$ & $B D_{6}$ & $\mathbb{Z}_{2} \times \mathbb{Z}^{2}$ \\
5 & 3 & $C_{3,1}, C_{3,2}$ & 3 & $D_{6}$ & $D_{2,12,5}$ & $\mathbb{Z}_{2}^{2} \times \mathbb{Z}^{2}$ \\
6 & 3 & $2 C_{2,1}$ & 2 & $A_{4} \times \mathbb{Z}_{2}$ & $\mathrm{G}(48,30)$ & $\mathbb{Z}_{2} \times \mathbb{Z}^{2}$ \\
6 & 7 & $2 C_{2,1}$ & 2 & $A_{4} \times \mathbb{Z}_{2}$ & $A_{4} \times \mathbb{Z}_{4}$ & $\mathbb{Z}_{2} \times \mathbb{Z}^{2}$ \\
6 & 5 & $C_{5,3}$ & 5 & $D_{5}$ & $\mathrm{G}(20,3)$ & $\mathbb{Z}_{2} \times \mathbb{Z}^{2}$ \\
8 & 5 & $\emptyset$ & $2^{2}$ & $\mathbb{Z}_{2} \times \mathbb{Z}_{4}$ & $D_{2,8,5}$ & $\mathbb{Z}_{4} \times \mathbb{Z}^{2}$ \\
8 & 5 & $\emptyset$ & $2^{2}$ & $D_{4}$ & $D_{2,8,3}$ & $\mathbb{Z}_{4} \times \mathbb{Z}^{2}$ \\
8 & 5 & $\emptyset$ & $2^{2}$ & $\mathbb{Z}_{2}^{3}$ & $\mathbb{Z}_{2}^{2} \rtimes \mathbb{Z}_{4}$ & $\mathbb{Z}_{2}^{3} \times \mathbb{Z}^{2}$ \\
\hline
\end{tabular}

Table 3. Mixed q.e. surfaces of general type with

\begin{tabular}{lccccc}
\multicolumn{7}{c}{$p_{g}=q=2$} \\
\cline { 1 - 5 }$K_{S}^{2}$ & $\mathcal{B}(X)$ & Sign. & $G^{0}$ & $G$ & $H_{1}(S, \mathbb{Z})$ \\
\hline 8 & $\emptyset$ & - & $\mathbb{Z}_{2}$ & $\mathbb{Z}_{4}$ & $\mathbb{Z}_{2} \times \mathbb{Z}^{4}$ \\
\hline
\end{tabular}

In Section 1, we give the algebraic recipe, which, using the Riemann's Existence Theorem, constructs mixed q.e. surfaces.

In Section 2 we give a complete description of the analytic type of the possible singularities of $X$. Moreover, we show how to compute the number of singular points of $X$ and the analytic type of each singularity directly by the ingredients of the above algebraic recipe, and we give formulas for $K_{S}^{2}, p_{g}(S)$ and $q(S)$. We think it is worth mentioning here an unexpected consequence of those formulas (Corollary 2.20): The number of branch points of the double cover $(C \times C) / G^{0} \rightarrow(C \times C) / G$ is even and bounded above by $2\left(p_{g}(S)+1\right)$.

Section 3 is devoted to the Albanese map of a mixed q.e. surface with $q=1$. The main result is a formula to compute the genus of its general fibre.

In Section 4, we show that all irregular mixed q.e. surfaces are minimal. In the regular case, we prove it under a strong assumption on the singularities of $X$ (Proposition 4.9).

Finally, in Section 5, we present our algorithm to construct all mixed quasi-étale surfaces with given values of $K^{2}, p_{g}$ and $q$, and prove Theorems A, B and C.

1. The algebraic recipe. Throughout this paper we will denote by $C$ a Riemann surface of genus $g \geq 2$ and by $G$ a finite subgroup of $\operatorname{Aut}(C \times C)$ whose action is free outside a finite subset and mixed, which means that there are elements in $G$ which 
exchange the two natural isotrivial fibrations of $C \times C$. We will denote by $G^{0}$ the index 2 subgroup consisting of those elements that do not exchange the factors.

We will say that the quotient surface $X=(C \times C) / G$ is a mixed q.e. quotient. We will denote by $\rho: S \rightarrow X$ the minimal resolution of the singularities of $X$, and we say that $S$ is a mixed q.e. surface.

REMARK 1.1. By [24, Remark 2.3] every mixed q.e. quotient is induced by a unique minimal action, which means that $G^{0}$ acts faithfully on both factors: therefore in this paper we will only consider minimal actions. If $X$ is a mixed q.e. quotient, then the quotient map factors as follows:

$$
C \times C \stackrel{\sigma}{\longrightarrow} Y:=(C \times C) / G^{0} \stackrel{\pi}{\longrightarrow} X .
$$

In [16, Proposition 3.16], the author gives the following description of minimal mixed actions.

Theorem 1.2. Let $G \subseteq \operatorname{Aut}(C \times C)$ be a minimal mixed action. Fix $\tau^{\prime} \in G \backslash G^{0}$; it determines an element $\tau:=\tau^{\prime 2} \in G^{0}$ and an element $\varphi \in \operatorname{Aut}\left(G^{0}\right)$ defined by $\varphi(h):=$ $\tau^{\prime} h \tau^{\prime-1}$. Then, up to a coordinate change, $G$ acts as follows:

$$
\begin{aligned}
g(x, y) & =(g x, \varphi(g) y) \\
\tau^{\prime} g(x, y) & =(\varphi(g) y, \tau g x) \quad \text { for } g \in G^{0} .
\end{aligned}
$$

Conversely, for every $G^{0} \subseteq \operatorname{Aut}(C)$ and $G$ extension of degree 2 of $G^{0}$, fixed $\tau^{\prime} \in$ $G \backslash G^{0}$ and $\tau$ and $\varphi$ defined as above, (1.1) defines a minimal mixed action on $C \times C$.

We recall the following results.

THEOREM 1.3 [24, Theorem 2.6]. Let $X$ be a quotient surface of mixed type provided by a minimal mixed action of $G$ on $C \times C$. The quotient map $C \times C \rightarrow X$ is quasi-étale if and only if the exact sequence

$$
1 \longrightarrow G^{0} \longrightarrow G \longrightarrow \mathbb{Z}_{2} \longrightarrow 1
$$

does not split.

Moreover, if the quotient map is quasi-étale, then $\operatorname{Sing}(X)=\pi(\operatorname{Sing}(Y))$.

LeMma 1.4 [24, Lemma 2.9]. Let $S \rightarrow X=(C \times C) / G$ be a mixed q.e. surface. Then $q(S)$ equals the genus of $C^{\prime}:=C / G^{0}$.

The study of varieties birational to a quotient of a product of curves is strictly connected with the study of Galois coverings of Riemann surfaces. Now we collect some results that allow us to shift from the geometrical setup to the algebraic one and vice versa.

DeFINITION 1.5. Let $H$ be a finite group and let

$$
g \geq 0 \quad \text { and } \quad m_{1}, \ldots, m_{r}>1
$$

be integers. A generating vector for $H$ of signature $\left(g ; m_{1}, \ldots, m_{r}\right)$ is a $(2 g+r)$-tuple of elements of $H$ :

$$
V:=\left(d_{1}, e_{1}, \ldots, d_{g}, e_{g} ; h_{1}, \ldots, h_{r}\right)
$$


such that $V$ generates $H, \prod_{i=1}^{g}\left[d_{i}, e_{i}\right] \cdot h_{1} \cdot h_{2} \cdots h_{r}=1$ and there exists a permutation $\sigma \in \mathfrak{S}_{r}$ such that $\operatorname{ord}\left(h_{i}\right)=m_{\sigma(i)}$ for $i=1, \ldots, r$. In this case, we also say that $H$ is $\left(g ; m_{1}, \ldots, m_{r}\right)$-generated.

By the Riemann's Existence Theorem (see [8]), any curve $C$ of genus $g$ together with an action of a finite group $H$ on it, such that $C / H$ is a curve $C^{\prime}$ of genus $g^{\prime}$, is determined (modulo automorphisms) by the following data:

(1) the branch point set $\left\{p_{1}, \ldots, p_{r}\right\} \subset C^{\prime}$;

(2) loops $\alpha_{1}, \ldots, \alpha_{g^{\prime}}, \beta_{1}, \ldots, \beta_{g^{\prime}}, \gamma_{1}, \ldots, \gamma_{r} \in \pi_{1}\left(C^{\prime} \backslash\left\{p_{1}, \ldots, p_{r}\right\}\right)$, where $\left\{\alpha_{i}, \beta_{i}\right\}_{i}$ generates $\pi_{1}\left(C^{\prime}\right)$, each $\gamma_{i}$ is a simple geometric loop around $p_{i}$ and $\prod_{i=1}^{g^{\prime}}\left[\alpha_{i}, \beta_{i}\right]$. $\gamma_{1} \cdot \ldots \cdot \gamma_{r}=1$

(3) a generating vector for $H$ of signature $\left(g^{\prime} ; m_{1}, \ldots, m_{r}\right)$ with the property that the Hurwitz's formula holds:

$$
2 g-2=|H|\left(2 g^{\prime}-2+\sum_{i=1}^{r} \frac{m_{i}-1}{m_{i}}\right) .
$$

Remark 1.6. Analogously, a mixed q.e. quotient $X=(C \times C) / G$ determines a finite group $G^{0}$, a degree 2 extension $1 \rightarrow G^{0} \rightarrow G \rightarrow \mathbb{Z}_{2} \rightarrow 1$, the curve $C^{\prime}=C / G^{0}$, a set of points $\left\{p_{1}, \ldots, p_{r}\right\} \subset C^{\prime}$, and, for every choice of $\alpha_{i}, \beta_{j}, \gamma_{k} \in \pi_{1}\left(C^{\prime} \backslash\left\{p_{1}, \ldots, p_{r}\right\}\right)$ as in (2), a generating vector $V$ for $G^{0}$.

Conversely, the following algebraic data:

- a finite group $G^{0}$;

- a curve $C^{\prime}$;

- points $p_{1}, \ldots, p_{r} \in C^{\prime}$, and $\alpha_{i}, \beta_{j}, \gamma_{k} \in \pi_{1}\left(C^{\prime} \backslash\left\{p_{1}, \ldots, p_{r}\right\}\right)$ as in (2);

- integers $m_{1}, \ldots, m_{r}>1$;

- a generating vector $V$ for $G^{0}$ of signature $\left(g\left(C^{\prime}\right) ; m_{1}, \ldots, m_{r}\right)$;

- a degree 2 extension $1 \rightarrow G^{0} \rightarrow G \rightarrow \mathbb{Z}_{2} \rightarrow 1$ which does not split;

give a uniquely determined mixed q.e. quotient. Indeed, by the Riemann's Existence Theorem the first five data give the Galois cover $c: C \rightarrow C / G^{0} \cong C^{\prime}$ branched over $\left\{p_{1}, \ldots, p_{r}\right\}$. The last datum determines, by Theorem 1.2, a minimal mixed action on $C \times C$ and by Theorem 1.3 the action is free outside a finite set of points.

If $V:=\left(d_{1}, e_{1}, \ldots, d_{g}, e_{g} ; h_{1}, \ldots, h_{r}\right)$, we will denote by $K_{i}$ the cyclic subgroup of $G^{0}$ generated by $h_{i}$.

2. The singularities of a mixed q.e. quotient. This section is devoted to the study of the singularities of a mixed q.e. quotient $X=(C \times C) / G$. We will need to consider the intermediate quotient $Y=(C \times C) / G^{0}$, and the two isotrivial fibrations $\alpha_{i}: Y \rightarrow$ $C^{\prime}=C / G^{0}$ induced by the projections of $C \times C$ on the two factors.

The double cover $\pi: Y \rightarrow X$ determines an involution $\iota: Y \rightarrow Y$ such that $X=$ $Y / \iota$. By the last statement of Theorem 1.3, the fixed points of $\iota$ are singularities of $Y$, hence $\iota$ splits the singularities of $X$ in two classes: the singularities not in the branch locus of $\pi$ (analytically isomorphic to each of its pre-images in $Y$ ), and the images of the fixed points of $\iota$. We then need to consider the singularities of $Y$ and the action of $\iota$ on them.

$Y$ is an unmixed quotient whose singularities are now well understood (see [9, 28, 34]). These are cyclic quotient singularities, isomorphic to the quotient $\mathbb{C}^{2} /\langle\sigma\rangle$, where $\sigma$ is the diagonal linear automorphism with eigenvalues $\exp \left(\frac{2 \pi i}{n}\right)$ and $\exp \left(\frac{2 \pi i a}{n}\right)$ 
with $n>a>0$ and $\operatorname{gcd}(a, n)=1$. We will say that this is a singularity of type $C_{n, a}$. Two singularities of respective types $C_{n, a}$ and $C_{n^{\prime}, a^{\prime}}$ are locally analytically isomorphic if and only if $n=n^{\prime}$ and either $a=a^{\prime}$ or $a a^{\prime} \equiv 1 \bmod n$. We read from [9] how to determine the singular points of $Y$ and their respective $n$ and $a$.

Proposition 2.1 [9, Propositions 1.16 and 1.18]. Let $X$ be a mixed q.e. quotient given by data as in Remark 1.6, let $Y=(C \times C) / G^{0}$ be the intermediate product-quotient surface, and consider the induced map $Q=\left(\alpha_{1}, \alpha_{2}\right): Y \rightarrow C^{\prime} \times C^{\prime}$. The singular points of $Y$ are the points $y=\sigma(u, v)$ such that

$$
\operatorname{Stab}_{G^{0}}(u) \cap \varphi^{-1}\left(\operatorname{Stab}_{G^{0}}(v)\right) \neq\{1\},
$$

where $\varphi$ is the automorphism of $G^{0}$ in Theorem 1.2. In particular, if $y \in \operatorname{Sing}(Y)$, then $Q(y)=\left(p_{i}, p_{j}\right)$ for some $i, j$. Now fix $i, j \in\{1, \ldots, r\}$, then

(i) there is a $G^{0}$-equivariant bijection $(Q \circ \sigma)^{-1}\left(p_{i}, p_{j}\right) \rightarrow G^{0} / K_{i} \times G^{0} / K_{j}$, where the action on the target is $g\left(a K_{i}, b K_{j}\right)=\left(g a K_{i}, \varphi(g) b K_{j}\right)$;

(ii) there is a $K_{i}$-equivariant bijection between the orbits of the above $G^{0}$-action on $G^{0} / K_{i} \times G^{0} / K_{j}$ with the orbits of the $K_{i}$-action on $\{\overline{1}\} \times G^{0} / K_{j}$.

(iii) An element $[g] \in\{\overline{1}\} \times G^{0} / K_{j}$ corresponds to a point of type $C_{n, a}$ on $Y$, where $n=\left|K_{i} \cap \varphi^{-1}\left(g K_{j} g^{-1}\right)\right|$, and $a$ is given as follows: let $\delta_{i}$ be the minimal positive integer such that there exists $1 \leq \gamma_{j} \leq \operatorname{ord}\left(h_{j}\right)$ with $h_{i}^{\delta_{i}}=g \varphi^{-1}\left(h_{j}^{\gamma_{j}}\right) g^{-1}$. Then $a=$ $\frac{n \gamma_{j}}{\operatorname{ord}\left(h_{j}\right)}$.

By Proposition 2.1 we can compute the singularities of $Y$ from the algebraic data of Remark 1.6. In order to compute the basket of singularities of $X$, we first need to know which of these are ramification points for $\pi$.

Lemma 2.2 [24, Proposition 3.8]. Let $y \in Y$ be a fixed point for $\iota$. Then $Q(y)=\left(p_{i}, p_{i}\right)$ for some $i$. In other words, $Q$ maps all fixed points of $\iota$ to the diagonal of $C^{\prime} \times C^{\prime}$.

Proposition 2.3. An element $[g] \in\{\overline{1}\} \times G^{0} / K_{i}$ corresponds to a fixed point for $\mathrm{c}$ if and only if there exists an element $h \in G^{0}$ such that:

$$
\left\{\begin{array}{l}
\varphi(h) \tau h \in K_{i} \\
\varphi(h) g \in K_{i}
\end{array} .\right.
$$

Proof. Point $\left(K_{i}, g K_{i}\right)$ corresponding to $[g]$ is a ramification point for $\pi$ if and only if there exists an element $\tau^{\prime} h \in G \backslash G^{0}$ such that $\left(K_{i}, g K_{i}\right)=\tau^{\prime} h\left(K_{i}, g K_{i}\right)=$ $\left(\varphi(h) g K_{i}, \tau h K_{i}\right)$, that is

$$
\left\{\begin{array} { l } 
{ \varphi ( h ) g K _ { i } = K _ { i } } \\
{ g K _ { i } = \tau h K _ { i } }
\end{array} \Longleftrightarrow \left\{\begin{array}{l}
\varphi(h) g K_{i}=K_{i} \\
\varphi(h) \tau h K_{i}=\left(\tau^{\prime} h\right)^{2} K_{i}=K_{i}
\end{array}\right.\right.
$$

We now study the action of $\iota$ on the neighbourhood of a singular point of $Y$. We denote by $\lambda: T \rightarrow Y$ the minimal resolution of the singularities of $Y$. The exceptional divisor $E$ of the minimal resolution of a cyclic quotient singularities of type $C_{n, a}$ is a Hirzebruch-Jung string, that is, $E=\sum_{i=1}^{l} E_{i}$, where all $E_{i}$ are smooth rational curves with $E_{i} \cdot E_{i+1}=1, E_{i} \cdot E_{j}=0$ for $|i-j| \geq 2$ and $E_{i}^{2}=-b_{i}$, where the integers $b_{i}$ are the 
coefficients of the continued fraction of $\frac{n}{a}$ :

$$
\frac{n}{a}=b_{1}-\frac{1}{b_{2}-\frac{1}{b_{3}-\ldots}}=:\left[b_{1}, \ldots, b_{l}\right] .
$$

REMARK 2.4. $a \cdot a^{\prime} \equiv 1 \bmod n$ if and only if the continued fraction of $\frac{n}{a^{\prime}}$ is $\left[b_{l}, \ldots, b_{1}\right]$.

We need the following.

LeMmA 2.5. The involution ı on $Y$ lifts to a morphism $\mu: T \rightarrow T$.

Proof. Consider $\mu:=\lambda^{-1} \circ \iota \circ \lambda: T \rightarrow T$. Let $\Gamma \subset T \times T$ be the graph of $\mu$; let $f_{1}, f_{2}: \Gamma \rightarrow T$ be the projections on the factors.

If $\mu$ is not defined at a point $p \in T$, then $\Gamma$ contains a $(-1)$-curve $C$ contracted to $p$ by $f_{1} . D:=f_{2}(C) \subset T$ is a curve contracted to $\iota(\lambda(p))$ by $\lambda$, so a component of a Hirzebruch-Jung string: in particular $D^{2} \leq-2$. On the other hand, since $f_{2}$ is a birational morphism, $D^{2} \geq C^{2}=-1$, a contradiction.

To study the action of $\mu$ on the Hirzebruch-Jung strings, we will need the following.

Proposition 2.6 (see [39, Theorem 2.1]). Let $y \in Y$ be a singular point of type $C_{n, a}$, and consider the two fibres $F_{1}:=\alpha_{1}^{*}\left(\alpha_{1}(y)\right)$ and $F_{2}:=\alpha_{2}^{*}\left(\alpha_{2}(y)\right)$ taken with the reduced structure. Let $\tilde{F}_{i}:=\lambda_{*}^{-1}\left(F_{i}\right)$ be the strict transforms of $F_{i}(i=1,2)$ and let $E$ be the exceptional divisor of $y$.

Then $\tilde{F}_{1}$ intersects one of the extremal curves of $E$, say $E_{1}$, while $\tilde{F}_{2}$ intersects the other extremal curve, say $E_{l}$.

Proposition 2.6 motivates the following.

Definition 2.7. Let $\alpha: Y \rightarrow C^{\prime}$ be one of the two natural fibrations. Let $y \in \operatorname{Sing}(Y)$ be a point of type $C_{n, a}$. Let $E:=\sum_{i=1}^{l} E_{i}$ be the exceptional divisor over $y$, where all $E_{i}$ are rational curves ordered so that $E_{i}^{2}=-b_{i}, E_{i} \cdot E_{i+1}=1$. Let $\tilde{F}$ be the strict transform in $T$ of fibre $F=\alpha^{*}(\alpha(y))$ taken with the reduced structure.

We say that $y$ is of type $C_{n, a}$ with respect to $\alpha$ if $\tilde{F}$ intersects $E_{1}$.

REMARK 2.8. If $y$ is of type $C_{n, a}$ with respect to $\alpha_{1}$, then $y$ is of type $C_{n, a^{\prime}}$ with respect to $\alpha_{2}$, with $a \cdot a^{\prime} \equiv 1 \bmod n$.

LEMMA 2.9. If $y$ is a point of type $C_{n, a}$ with respect to $\alpha_{1}$, then $\iota(y)$ is a point of type $C_{n, a^{\prime}}$ with respect to $\alpha_{1}$, with $a \cdot a^{\prime} \equiv 1 \bmod n$.

Proof. Since $\iota$ is an isomorphism, $y$ and $z:=\iota(y)$ have the same analytic type, so $z$ is either of type $C_{n, a}$ or type $C_{n, a^{\prime}}$ with respect to $\alpha_{1}$.

Let $Y_{i}$, resp. $Z_{i}$ be the fibre of $\alpha_{i}$ containing $y$, resp. $z$, all of them taken with the reduced structure, and let $\tilde{Y}_{i}:=\lambda_{*}^{-1}\left(Y_{i}\right)$ and $\tilde{Z}_{i}:=\lambda_{*}^{-1}\left(Z_{i}\right)(i=1,2)$ be their strict transforms in $T$. Note that $\iota$ is the map induced on $Y$ by the action on $C \times C$ of any $\tau^{\prime} \in G \backslash G^{0}$. Since $\tau^{\prime}$ exchanges the two factors, then $\iota\left(Y_{1}\right)=Z_{2}$ and therefore $\mu\left(\tilde{Y}_{1}\right)=\tilde{Z}_{2}$.

Let $E=\sum_{i=1}^{l} E_{i}$ resp. $E^{\prime}=\sum_{i=1}^{l} E_{i}^{\prime}$ be the exceptional divisor of $y$ resp. $z$, with the $E_{i}$ resp. $E_{i}^{\prime}$ ordered as in Definition 2.7 for $\alpha=\alpha_{1}$. By assumption, $\tilde{Y}_{1}$ intersects $E_{1}, \tilde{Z}_{1}$ intersects $E_{1}^{\prime}, \tilde{Z}_{2}$ intersects $E_{l}^{\prime}$.

Since $\mu\left(\tilde{Y}_{1}\right)=\tilde{Z}_{2}$, then $\mu\left(E_{1}\right)=E_{l}^{\prime}$. It follows that $z$ is of type $C_{n, a}$ with respect to $\alpha_{2}$ and of type $C_{n, a^{\prime}}$ with respect to $\alpha_{1}$. 

of $\iota$.

We now give a full description of the singular points of $X$ arising from fixed points

Proposition 2.10. Let $X=(C \times C) / G$ be a mixed q.e. quotient, and let $y \in Y$ be a fixed point of $\iota$. Then $y$ is a singularity of type $C_{n, a}$ with $a^{2} \equiv 1 \bmod n$; so $a=a^{\prime}$ and the continued fraction $\frac{n}{a}=\left[b_{1}, \ldots, b_{l}\right]$ is palindromic: $b_{i}=b_{l+1-i} \forall i$.

Moreover,

(i) $n$ is even;

(ii) $l$ is odd: $l=2 m+1$ and $b_{m+1}$ is even;

(iii) the exceptional divisor of the minimal resolution of the singular point $\pi(y)$ is a tree of $m+3$ smooth rational curves with decorated dual graph:

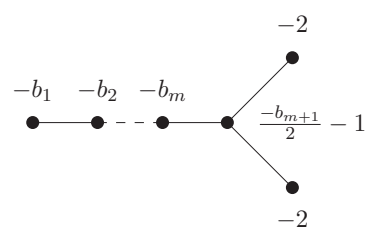

Proof. By Lemma 2.9, $y$ is of type $C_{n, a}$ with respect to both $\alpha_{j}$, so $a=a^{\prime}$ and $b_{i}=b_{l+1-i}$. More precisely, the proof of Lemma 2.9 shows that if $E=\sum_{1}^{l} E_{i}$ is the HirzebruchJung string over $y, \mu\left(E_{i}\right)=E_{l+1-i}$.

(i) If $y=\sigma(u, v),\left|\operatorname{Stab}_{G^{0}}(u, v)\right|=n$ and $\left|\operatorname{Stab}_{G}(u, v)\right|=2 n$. If $n$ is odd, then by Sylow's theorem there exists an element $g$ of order 2 in $\operatorname{Stab}_{G}(u, v) \backslash \operatorname{Stab}_{G^{0}}(u, v)$, splitting the exact sequence (1.2), a contradiction.

(ii) Let $D=\sum_{i=1}^{l} D_{i}:=\lambda^{-1}(y)$, and assume that $l=2 m$ is even. The involution $\mu$ exchanges $D_{i}$ with $D_{l+1-i}$, hence $p=D_{m} \cap D_{m+1}$ is the unique point of $D$ fixed by $\mu$. $\mathrm{d} \mu_{p}$ exchanges the directions of the tangent spaces of $D_{m}$ and $D_{m+1}$ and therefore it is not a multiple of the identity. Since it is an involution, then up to a linear coordinate change $\mathrm{d} \mu_{p}=\left(\begin{array}{cc}-1 & 0 \\ 0 & 1\end{array}\right)$, which implies that the fixed locus of $\mu$ contains a curve through $p$ in the direction of the eigenspace with eigenvalue 1 , a contradiction. We delay the proof that $b_{m+1}$ is even.

(iii) By part (ii), $l=2 m+1$ and all fixed points of $\mu$ in $D$ belong to $D_{m+1}=$ $\mu\left(D_{m+1}\right)$. The restriction of $\mu$ to $D_{m+1}$ is an involution and therefore by the Hurwitz's formula it fixes exactly two points $p_{1}$ and $p_{2}$ that are distinct from the points of intersection of $D_{m+1}$ with $D_{m}$ or $D_{m+2}$.

Let $V$ be a small $\mu$-invariant open set of $T$ containing $D$ and not intersecting any other exceptional divisor so that $\lambda(V)$ is an open set of $Y$ containing only one singular point: $y$. Let $\epsilon: V^{\prime} \rightarrow V$ be the blowup in $p_{1}$ and $p_{2}$, we denote by $D_{i}^{\prime}$ the strict transform of $D_{i}$, and by $A_{1}$ and $A_{2}$ the two (-1)-exceptional curves. The involution $\mu$ lifts to an involution $\mu^{\prime}$ on $V^{\prime}$ whose fixed locus is the smooth curve $A_{1} \cup A_{2}$.

Then $V^{\prime} / \mu^{\prime}$ is smooth, and therefore is a resolution of singular point $\pi(x)$ whose exceptional divisor is $D / \mu$. The computation of the dual graph of $D / \mu$ is a standard computation that we leave to the reader. We note that there is no curve with selfintersection -1 , so the resolution is the minimal resolution. Moreover, there is a curve of self-intersection $-\left(1+b_{m+1} / 2\right)$, showing that $b_{m+1}$ is even.

It follows that the analytic type of a singularity on $X$ only depends on its pre-image on $Y$. Indeed, these quotient singularities can be described as follows. 
Proposition 2.11. Let $X=(C \times C) / G$ be a mixed q.e. quotient, and let $y \in \operatorname{Sing}(Y)$ be a point of type $C_{n, a}$ with $\frac{n}{a}=\left[b_{1}, \ldots, b_{m}, 2 b, b_{m}, \ldots, b_{1}\right]$. Let

$$
\frac{p}{q}:=\left[b_{1}, \ldots, b_{m}\right], \quad \text { and } \quad \xi:=b p-q
$$

If $y$ is a ramification point for $\pi$, then $x:=\pi(y)$ is a quotient singularity isomorphic to $\mathbb{C}^{2} / H$ with:

- if $\xi=0$ (i.e. $p=0$ ), then

$$
H=\left\langle\left(\begin{array}{cc}
\epsilon & 0 \\
0 & \epsilon^{n+1}
\end{array}\right)\right\rangle, \quad \text { with } \epsilon=e^{\frac{2 \pi i}{2 n}},
$$

- if $\xi \neq 0$ and odd, then

$$
H=\left\langle\left(\begin{array}{ll}
\eta & 0 \\
0 & \eta
\end{array}\right),\left(\begin{array}{cc}
\omega & 0 \\
0 & \omega^{-1}
\end{array}\right),\left(\begin{array}{cc}
0 & 1 \\
-1 & 0
\end{array}\right)\right\rangle, \text { with } \eta=e^{\frac{2 \pi i}{2 \xi}}, \omega=e^{\frac{2 \pi i}{2 p}}
$$

- if $\xi \neq 0$ and even, then

$$
H=\left\langle\left(\begin{array}{cc}
0 & \zeta \\
-\zeta & 0
\end{array}\right),\left(\begin{array}{cc}
\omega & 0 \\
0 & \omega^{-1}
\end{array}\right)\right\rangle \text {, with } \zeta=e^{\frac{2 \pi i}{4 \xi}} \text { and } \omega=e^{\frac{2 \pi i}{2 p}}
$$

Proof. The statement follows immediately from the classification of finite subgroups of $\operatorname{GL}(2, \mathbb{C})$ without quasi-reflections, see [14, Satz 2.11] or [27, Theorem 4.6.20].

Definition 2.12. We say that a singular point $x$ as in Proposition 2.11 is a singular point of type $D_{n, a}$.

REMARK 2.13.

(1) A singular point of type $D_{n, a}$ is a Rational Double Point if and only if $a=n-1$, in which case we have a Rational Double Point of type $D_{\frac{n}{2}+2}$ (if $n=2$, this is more commonly known as $A_{3}$ ).

(2) A singular point of type $D_{n, a}$ is a cyclic quotient singularity if and only if $a=1$. More precisely, singularities of type $D_{n, 1}$ are isomorphic to singularities of type $C_{2 n, n+1}$. We will distinguish between these, keeping track of the branching locus of $\pi$.

(3) We noted that a point of type $C_{n, a}$ is also a point of type $C_{n, a^{\prime}}$ with $a^{\prime}=a^{-1}$ in $\mathbb{Z}_{n}$. We consider these different representations as equal and usually we do not distinguish between these.

In the following, the term multiset will be used in the sense of MAGMA [26]. So a multiset is a set whose elements have a multiplicity: a positive integer; the cardinality of a multiset takes into account the multiplicity of its elements.

Definition 2.14. Let $Y$ be an unmixed quotient. Then we define the basket of singularities of $Y$ to be the multiset

$$
\mathcal{B}(Y):=\left\{\lambda \times C_{n, a}: Y \text { has exactly } \lambda \text { singularities of type } C_{n, a}\right\} .
$$


Let $X=(C \times C) / G$ be a mixed q.e. quotient. We define the following two multisets:

$$
\begin{array}{r}
\mathcal{B}_{C}:=\left\{\eta \times C_{n, a}: X \text { has exactly } \eta \text { singularities of type } C_{n, a}\right. \\
\text { not in the branch locus of } \pi\} . \\
\mathcal{B}_{D}:=\left\{\zeta \times D_{m, b}: X \text { has exactly } \zeta \text { singularities of type } D_{m, b}\right. \\
\text { in the branch locus of } \pi\} .
\end{array}
$$

DEFINITION 2.15. The basket of singularities of $X$ is the multiset

$$
\mathcal{B}(X)=\mathcal{B}_{C} \cup \mathcal{B}_{D}
$$

The following is a useful constraint on the basket of singularities.

Proposition 2.16. Let $X=(C \times C) / G$ be a mixed q.e. quotient. Let $\mathcal{B}(X)=\mathcal{B}_{C} \cup \mathcal{B}_{D}$ be the basket of singularities of $X$ with $\mathcal{B}_{C}:=\left\{\eta_{i} \times C_{n_{i}, a_{i}}\right\}_{i}$ and $\mathcal{B}_{D}:=\left\{\zeta_{j} \times D_{m_{j}, b_{j}}\right\}_{j}$. Then

$$
\sum_{i} \eta_{i} \frac{a_{i}+a_{i}^{\prime}}{n_{i}}+\sum_{j} \zeta_{j} \frac{b_{j}}{m_{j}} \in \mathbb{Z}
$$

Proof. If $x \in X$ is a singular point of type $C_{n, a}$, then by Lemma $2.9 \pi^{-1}(x)$ is given by two singular points, one of type $C_{n, a}$ with respect to $\alpha_{1}$ and the other of type $C_{n, a^{\prime}}$ with respect to $\alpha_{1}$. If $x \in X$ is a singular point of type $D_{m, b}$, then $\pi^{-1}(x)$ is given by a unique singular point of type $C_{m, b}$ with respect to $\alpha_{1}$. The result now follows directly from [34, Proposition 2.8].

Definition 2.17. Let $x$ be a singular point of type $C_{n, a}$ with $\frac{n}{a}:=\left[b_{1}, \ldots, b_{l}\right]$. We define the following non-negative rational numbers:
(i) $k_{x}=k\left(C_{n, a}\right):=-2+\frac{2+a+a^{\prime}}{n}+\sum_{i=1}^{l}\left(b_{i}-2\right)$;
(ii) $e_{x}=e\left(C_{n, a}\right):=l+1-\frac{1}{n} \geq 0$.

Let $x$ be a singular point of type $D_{n, a}$ with $\frac{n}{a}:=\left[b_{1}, \ldots, b_{m}, 2 b, b_{m}, \ldots, b_{1}\right]$. We define the analogous non-negative rational numbers
(i) $k_{x}=k\left(D_{n, a}\right):=\frac{k\left(C_{n, a}\right)}{2}=-2+\frac{a+1}{n}+\sum_{i=1}^{m}\left(b_{i}-2\right)+b$;
(ii) $e_{x}=e\left(D_{n, a}\right):=\frac{e\left(C_{n, a}\right)}{2}+3=m+4-\frac{1}{2 n}$.

In both cases we set $B_{x}:=2 e_{x}+k_{x}$. Note that $B\left(D_{n, a}\right)=\frac{B\left(C_{n, a}\right)}{2}+6$.

Let $\mathcal{B}$ be a basket of singularities. We use the following notation:

$$
k(\mathcal{B})=\sum_{x \in \mathcal{B}} k_{x}, \quad e(\mathcal{B})=\sum_{x \in \mathcal{B}} e_{x}, \quad B(\mathcal{B})=\sum_{x \in \mathcal{B}} B_{x}
$$

These correction terms determine the invariants of $S$ as follows. 
Proposition 2.18. Let $\rho: S \rightarrow X=(C \times C) / G$ be a mixed q.e. surface, and let $\mathcal{B}$ be the basket of singularities of $X$. Then,

$$
\begin{gathered}
K_{S}^{2}=\frac{8(g-1)^{2}}{|G|}-k(\mathcal{B}) ; \\
e(S)=\frac{4(g-1)^{2}}{|G|}+e(\mathcal{B}) .
\end{gathered}
$$

Proof. Since the quotient map $C \times C \rightarrow X$ is quasi-étale, we get

$$
K_{X}^{2}=\frac{K_{Y}^{2}}{2}=\frac{8(g-1)^{2}}{|G|} .
$$

Let $\mathcal{B}=\mathcal{B}_{C} \cup \mathcal{B}_{D}=\left\{\eta_{i} \times C_{n_{i}, a_{i}}\right\}_{i} \cup\left\{\zeta_{j} \times D_{n_{j}, a_{j}}\right\}_{j}$, then the basket of singularities of $Y$ is $\mathcal{B}(Y)=\left\{2 \eta_{i} \times C_{n_{i}, a_{i}}\right\}_{i} \cup\left\{\zeta_{j} \times C_{n_{j}, a_{j}}\right\}_{j}$, hence by definition $k(\mathcal{B}(Y))=2 k(\mathcal{B})$. By [6, Proposition 2.6], we get

$$
K_{T}^{2}=\frac{8(g-1)^{2}}{\left|G^{0}\right|}-k(\mathcal{B}(Y))
$$

Let $\epsilon: T^{\prime} \rightarrow T$ be the blow-up of $T$ in the $2 d\left(d=\left|\mathcal{B}_{D}\right|\right)$ points fixed by $\mu$ :

$$
K_{T^{\prime}}^{2}=K_{T}^{2}-2 d=K_{Y}^{2}-k(\mathcal{B}(Y))-2 d=2\left(K_{X}^{2}-k(\mathcal{B})-d\right) .
$$

By the proof of Proposition 2.10, we have a double cover $\tilde{\pi}: T^{\prime} \rightarrow S$ branched over $F:=F_{1}+\ldots+F_{2 d}$, where all $F_{i}$ are smooth rational curves with $F_{i}^{2}=-2$ and and $F_{i} . F_{j}=0$ if $i \neq j$. Then numerically [23, pp. 13-14] $K_{T^{\prime}}$ equals $\tilde{\pi}^{*}\left(K_{S}+F / 2\right)$ and, since $K_{S} \cdot F=0$, it follows:

$$
K_{T^{\prime}}^{2}=2\left(K_{S}+\frac{F}{2}\right)^{2}=2\left(K_{S}^{2}+\frac{-4 d}{4}\right)=2\left(K_{S}^{2}-d\right) .
$$

From equations (2.3) and (2.4), we get:

$$
K_{S}^{2}=K_{X}^{2}-k(\mathcal{B})=\frac{8(g-1)^{2}}{|G|}-k(\mathcal{B}) .
$$

Let $X^{0}:=X \backslash \operatorname{Sing}(X)$ be the smooth locus of $X$; arguing as in [6] we get:

$$
e(S)=e\left(X^{0}\right)+\sum_{x \in \mathcal{B}_{C}}\left(l_{x}+1\right)+\sum_{x \in \mathcal{B}_{D}}\left(m_{x}+4\right)
$$

and

$$
e\left(X^{0}\right)=\frac{e(C \times C)}{|G|}-\sum_{x \in \mathcal{B}_{C}} \frac{1}{n_{x}}-\sum_{x \in \mathcal{B}_{D}} \frac{1}{2 n_{x}} .
$$


It follows that

$$
e(S)=\frac{4(g-1)^{2}}{|G|}+e(\mathcal{B})
$$

Using Noether's formula and Proposition 2.18 we get the following.

Corollary 2.19. Let $\rho: S \rightarrow X=(C \times C) / G$ be a mixed q.e. surface, and let $\mathcal{B}$ be the basket of singularities of $X$. Then,

$$
K_{S}^{2}=8 \chi(S)-\frac{1}{3} B(\mathcal{B})
$$
$\mathcal{B}_{D}$

We conclude this section by showing a very strong restriction on the cardinality of

COROLlary 2.20. Let $\rho: S \rightarrow X=(C \times C) / G$ be a mixed q.e. surface. The cardinality $d$ of $\mathcal{B}_{D}$ is even and

$$
\frac{d}{2} \leq p_{g}(S)+1
$$

Corollary 2.20 follows from the next proposition since the singular points of $X$ of type $D_{n, a}$ are the branch points of $\pi$.

Proposition 2.21. Let $\rho: S \rightarrow X=(C \times C) / G$ be a mixed q.e. surface, and let $\lambda: T \rightarrow$ $Y$ be the minimal resolution of the singularities of $Y$. Let $d$ be the number of fixed points for $\iota$, then

$$
p_{g}(S) \leq p_{g}(T)=2 p_{g}(S)+1-\frac{d}{2} .
$$

Proof. Let $\epsilon: T^{\prime} \rightarrow T$ be the blowup of $T$ in the $2 d$ points fixed by $\mu$; we have a double cover $\tilde{\pi}: T^{\prime} \rightarrow S$ branched along $2 d$ smooth pairwise disjoint rational curves. Pulling back the forms on $S$ to forms on $T^{\prime}$ we note that $p_{g}(S) \leq p_{g}\left(T^{\prime}\right)=p_{g}(T)$. Moreover, $e\left(T^{\prime}\right)=2 e(S)-4 d, e(T)=e\left(T^{\prime}\right)-2 d=2 e(S)-6 d$ and $K_{T}^{2}=2 K_{S}^{2}$ by (2.3) and (2.4). By Noether's formula:

$$
\chi\left(\mathcal{O}_{T}\right)=\frac{1}{12}\left(K_{T}^{2}+e(T)\right)=\frac{1}{12}\left(2 K_{S}^{2}+2 e(S)-6 d\right)=2 \chi\left(\mathcal{O}_{S}\right)-\frac{d}{2} .
$$

Since $T \rightarrow Y$ is a product-quotient surface, $q(T)=2 g\left(C / G^{0}\right)=2 q(S)$ and

$$
p_{g}(T)=2+2 p_{g}(S)-2 q(S)-\frac{d}{2}+q(T)-1=2 p_{g}(S)+1-\frac{d}{2} .
$$

3. The Albanese fibre of a mixed q.e. surface with irregularity 1. The Albanese map of a surface of general type $S$ with irregularity 1 is a fibration onto the elliptic curve $\operatorname{Alb}(S)$. The genus $g_{a l b}$ of the general Albanese fibre is a deformation invariant, which is very important from the point of view of the geography of surfaces of general type. In this section we show how to compute $g_{a l b}$ for mixed q.e. surfaces. 
Let $S \stackrel{\rho}{\rightarrow} X=(C \times C) / G$ be a mixed q.e. surface with $q(S)=1$. By Lemma 1.4, $C^{\prime}=C / G^{0}$ is an elliptic curve, so in this section we will set $E:=C^{\prime}$. We have the following commutative diagram:

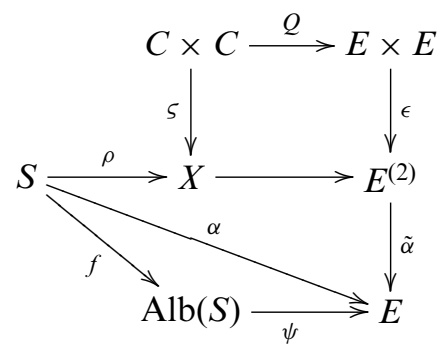

where $\tilde{\alpha}$ is the Abel-Jacobi map. By the properties of the Albanese torus (see [3, Proposition I.13.9]), the Stein factorization of $\alpha$ is given by the Albanese map $f: S \rightarrow$ $\operatorname{Alb}(S)$ and a (unique) homomorphism $\psi: \operatorname{Alb}(S) \rightarrow E$.

The Galois cover $c: C \rightarrow E$ has branching set $B:=\left\{p_{1}, \ldots, p_{r}\right\}$; up to translation we may assume that the neutral element 0 of $E$ is not in $B$, and that $-p_{i} \notin B$ for each $i \in\{1, \ldots, r\}$.

Let $E^{\prime}:=\epsilon^{*}\left(\tilde{\alpha}^{*}(0)\right)=\{(x,-x) \mid x \in E\} \cong E$, consider $F^{*}:=Q^{*}\left(E^{\prime}\right)$ and let $F:=$ $\alpha^{*}(0)$. Note that $\rho(F)=\varsigma\left(F^{*}\right)$. Our assumption $-p_{i} \notin B$ ensures that $F^{*}$ and $F$ are smooth, and the arithmetic genus of $F$ can be easily computed by the Hurwitz's formula, see equation (3.4) below. $F$ is the disjoint union of $\operatorname{deg} \psi$ fibres of the Albanese map, so to compute $g_{\text {alb }}$ we need to compute deg $\psi$ first.

We will need the points $q_{i}:=\left(p_{i},-p_{i}\right)$ and $q_{i}^{\prime}:=\left(-p_{i}, p_{i}\right)$ of $E^{\prime}$; we set $B^{\prime}:=\left\{q_{i}, q_{i}^{\prime}\right\}_{i}$. We note that $0^{\prime}=(0,0) \in E^{\prime} \backslash B^{\prime}$.

By Remark 1.6, given suitable loops $\alpha, \beta, \gamma_{1}, \ldots, \gamma_{r} \in \pi_{1}(E \backslash B, 0)$, the cover $c: C \rightarrow$ $E$ is determined by a generating vector $\left(a, b ; h_{1}, \ldots, h_{r}\right)$ of $G^{0}$, representing the monodromy map $\mu: \pi_{1}(E \backslash B, 0) \rightarrow G^{0}$ of $c$.

Since $Q=c \times c$, the monodromy map of the $G^{0} \times G^{0}$-cover $Q$ is given by two copies of $\mu$. $Q$ induces by restriction the $G^{0} \times G^{0}$-cover $F^{*} \rightarrow E^{\prime}$, whose branching locus is $B^{\prime}$. To describe its monodromy map, we choose generators $\delta, \theta, \gamma_{1}^{\prime}, \ldots, \gamma_{r}^{\prime}, \gamma_{1}^{\prime \prime}, \ldots, \gamma_{r}^{\prime \prime} \in \pi_{1}\left(E^{\prime} \backslash B^{\prime}, 0^{\prime}\right)$ as follows:

- $\delta=(\alpha,-\alpha)$,

- $\theta=(\beta,-\beta)$,

- $\gamma_{i}^{\prime}=\left(\gamma_{i},-\gamma_{i}\right)$ are geometric loops around $q_{i}$,

- $\gamma_{i}^{\prime \prime}=\left(-\gamma_{i}, \gamma_{i}\right)$ are geometric loops around $q_{i}^{\prime}$.

Please note that we need some care in the choice of loops $\alpha, \beta, \gamma_{i}$ to ensure that $\delta, \theta, \gamma_{i}^{\prime}$ and $\gamma_{i}^{\prime \prime}$ do not meet $B^{\prime}$.

Moreover, the class of $\delta, \theta, \gamma_{i}^{\prime}$ and $\gamma_{i}^{\prime \prime}$ in $\pi_{1}\left(E^{\prime} \backslash B^{\prime}, 0^{\prime}\right)$ depends on the choice of loops $\alpha, \beta, \gamma_{i}$ and not only on their class in $\pi_{1}(E \backslash B, 0)$. Anyway, the classes of $\delta, \theta, \gamma_{i}^{\prime}$ and $\gamma_{i}^{\prime \prime}$ generate $\pi_{1}\left(E^{\prime} \backslash B^{\prime}, 0^{\prime}\right)$ and the monodromy map of $Q_{\mid F^{*}}: F^{*} \rightarrow E^{\prime}$ is the unique homomorphism $\pi_{1}\left(E^{\prime} \backslash B^{\prime}, 0^{\prime}\right) \stackrel{\mu^{\prime}}{\longrightarrow} G^{0} \times G^{0}$ such that

$$
\begin{aligned}
\delta \stackrel{\mu^{\prime}}{\longmapsto}\left(a, a^{-1}\right), & \theta \stackrel{\mu^{\prime}}{\longmapsto}\left(b, b^{-1}\right), \\
\gamma_{i}^{\prime} \stackrel{\mu^{\prime}}{\longmapsto}\left(h_{i}, 1\right), & \gamma_{i}^{\prime \prime} \stackrel{\mu^{\prime}}{\longmapsto}\left(1, h_{i}\right) .
\end{aligned}
$$


REMARK 3.1. (1) We note that the index of $\operatorname{Im}\left(\mu^{\prime}\right)$ in $G^{0} \times G^{0}$ equals the number of connected components of $F^{*}$.

(2) Fixed $\tau^{\prime} \in G \backslash G^{0}$, let $\tau:=\tau^{\prime 2} \in G^{0}$ and $\varphi \in \operatorname{Aut}\left(G^{0}\right)$ defined by $\varphi(h):=\tau^{\prime} h \tau^{\prime-1}$. We define the following action of $G$ on $G^{0} \times G^{0}$ :

$$
\begin{aligned}
g\left(h_{1}, h_{2}\right) & =\left(g h_{1}, \varphi(g) h_{2}\right) \\
\tau^{\prime} g\left(h_{1}, h_{2}\right) & =\left(\varphi(g) h_{2}, \tau g h_{1}\right) \quad \text { for } g \in G^{0} .
\end{aligned}
$$

We define

$$
M:=\left|\bigcup_{g \in G} g \operatorname{Im}\left(\mu^{\prime}\right)\right|
$$

LEMMA 3.2. Let $S$ be a mixed q.e. surface with $q(S)=1$. Then $\operatorname{deg} \psi=\frac{\left|G^{0}\right|^{2}}{M}$.

Proof. Let $u \in E^{\prime}$. The action of $G^{0} \times G^{0}$ on $Q^{-1}(u)$ induces a bijection between $G^{0} \times$ $G^{0}$ and $Q^{-1}(u)$; two points of $Q^{-1}(u)$ belong to the same connected component of $F^{*}$ if and only if the corresponding elements in $G^{0} \times G^{0}$ differ by an element in $\operatorname{Im}\left(\mu^{\prime}\right)$.

Moreover, two points $h, h^{\prime} \in F^{*}$ map to the same point of $X$ if and only if there exists $g \in G$ such that $g\left(h^{\prime}\right)=h$. So exactly $M$ points of $Q^{-1}(u)$ are mapped into each connected component of $\varsigma\left(F^{*}\right)$. We conclude since deg $\psi$ equals the number of connected components of $F$.

Proposition 3.3. Let $S$ be a mixed q.e. surface with $q(S)=1$, then

$$
g_{a l b}=1+\frac{g(C)-1}{\left|G^{0}\right|^{2}} M .
$$

Proof. Let us look at diagram (3.1). Since $G^{0}$ is $\left(1 ; m_{1}, \ldots, m_{r}\right)$-generated, then $e(C)=-\left|G^{0}\right| \sum_{i=1}^{r}\left(\frac{m_{i}-1}{m_{i}}\right)$. The $\left(G^{0} \times G^{0}\right)$-cover $Q$ is branched exactly along the union of $r$ 'horizontal' copies of $E$ and $r$ 'vertical' copies of $E$; moreover, for each $i$ there are one horizontal copy and one vertical copy with branching index $m_{i}$. Since $E^{\prime}$ is an elliptic curve that intersects all these copies of $E$ transversally in one point, by the Hurwitz's formula applied to $F^{*} \rightarrow E^{\prime}$ we obtain

$$
e\left(F^{*}\right)=-\left|G^{0}\right|^{2} \sum_{i=1}^{r} 2\left(\frac{m_{i}-1}{m_{i}}\right) .
$$

On the other hand, the $G$-cover $\zeta$ is q.e. and we get

$$
e(F)=\frac{e\left(F^{*}\right)}{|G|}=-\left|G^{0}\right| \sum_{i=1}^{r}\left(\frac{m_{i}-1}{m_{i}}\right)=e(C) .
$$



therefore

By Lemma 3.2, $F$ is the disjoint union of $\operatorname{deg} \psi=\frac{\left|G^{0}\right|^{2}}{M}$ curves of genus $g_{a l b}$, and

$$
2-2 g(C)=e(F)=\frac{\left|G^{0}\right|^{2}}{M}\left(2-2 g_{a l b}\right) .
$$

4. The minimal model. In this section, we want to determine the minimal model of the surfaces we construct. We start by recalling some useful results.

Lemma 4.1 [13, Proposition 1]. On a smooth surface $S$ of general type, every irreducible curve $C$ satisfies $K_{S} . C \geq-1$.

LEMMA 4.2 [9, Remark 4.3]. On a smooth surface $S$ of general type, every irreducible curve $C$ with $K_{S} . C \leq 0$ is smooth and rational.

Proposition 4.3. Let $S$ be a smooth surface of general type. If $E$ and $C$ are distinct smooth rational curves with $E^{2}=-1, C^{2} \geq-4$, then $C . E \leq 1$.

Proof. Assume by contradiction $C . E \geq 2$. Let $b: S \rightarrow S^{\prime}$ be the blow-down given by the contraction of $E$ and set $C^{\prime}:=b(C)$. By the assumption $C . E \geq 2, C^{\prime}$ is singular, so by Lemma 4.2:

$$
\begin{aligned}
0<K_{S^{\prime}} \cdot C^{\prime} & =\left(K_{S}-E\right) \cdot(C+(C \cdot E) E) \\
& =\left(K_{S}-E\right) \cdot C \\
& =-C^{2}-2-E \cdot C \leq 0
\end{aligned}
$$

a contradiction.

COROLlary 4.4. Let $S$ be a smooth surface of general type. Assume that $E$ is a $(-1)$-curve on $S$, then $E$ intersects at most one (-2)-curve.

Proof. Suppose $E$ intersects two (-2)-curves. By Proposition 4.3, it intersects each of the $(-2)$-curves transversally in a point. Then contracting $E$ we get two $(-1)$-curves intersecting in a point, which is not possible on a surface of general type.

The following is the main result of this section, showing that in the irregular case, the surfaces obtained are automatically minimal.

THEOREM 4.5. Let $S$ be an irregular mixed q.e. surface of general type, then $S$ is minimal.

Proof. Aiming for a contradiction, let $E$ be a $(-1)$-curve on $S$.

Consider the intermediate quotient $Y=(C \times C) / G^{0}$, the minimal resolution of its singularities $\lambda: T \rightarrow Y$ and the involution $\mu$ on $T$ (Lemma 2.5). Let $\epsilon: T^{\prime} \rightarrow T$ be the blowup of the fixed points of $\mu$. By Proposition 2.10 and its proof, there is a map $\tilde{\pi}: T^{\prime} \rightarrow S$, which is a double cover ramified along the exceptional divisors of $\epsilon$, so branched along a disjoint union of $(-2)$-curves.

Since $E$ can intersect at most one $(-2)$-curve, then $\tilde{\pi}^{*}(E)$ is a union of two rational curves; let $R$ be one of them. By construction, $R$ is not exceptional for the resolution $T^{\prime} \rightarrow Y$, and therefore one of the fibrations $\alpha_{i}: Y \rightarrow C^{\prime}$ induces a surjective map from a rational curve to $C^{\prime}$, contradicting $g\left(C^{\prime}\right)=q(S)>0$. 
In the case $q=0$, we borrow an argument of [9]. Let $\Gamma \subset X=(C \times C) / G$ be a rational curve. Let $\Gamma^{\prime}:=(\pi \circ \sigma)^{*}(\Gamma)=\sum_{1}^{k} n_{i} \Gamma_{i}$ be the decomposition in irreducible components of its pull back to $C \times C$. We observe that, since $\pi \circ \sigma$ is quasi-étale, $\forall i$ $n_{i}=1$ for every $i$ and that $G$ acts transitively on the set $\left\{\Gamma_{i} \mid i=1, \ldots, k\right\}$. Hence, there is a subgroup $H \triangleleft G$ of index $k$ acting on $\Gamma_{1}$ such that $\pi\left(\sigma\left(\Gamma_{1}\right)\right)=\Gamma_{1} / H=\Gamma$.

Normalizing $\Gamma_{1}$ and $\Gamma$, we get the following commutative diagram:

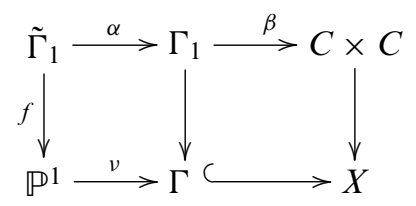

Since each automorphism lifts to the normalization, $H$ acts on $\tilde{\Gamma}_{1}$, and $f$ is the quotient map $\tilde{\Gamma}_{1} \rightarrow \tilde{\Gamma}_{1} / H \cong \mathbb{P}^{1}$. Moreover, $\beta\left(\alpha\left(\tilde{\Gamma}_{1}\right)\right)$ is a curve in $C \times C$, and therefore surjects on $C$, hence $g\left(\tilde{\Gamma}_{1}\right) \geq g(C) \geq 2$ and so $f$ is branched in at least three points.

LEMMA 4.6. Let $p$ be a branch point of $f$, then $v(p)$ is a singular point of $X$.

Proof. Let $p^{\prime} \in f^{-1}(p) \subset \tilde{\Gamma}_{1}$ be a ramification point of $f$, then $\operatorname{Stab}_{H}\left(p^{\prime}\right):=H_{1} \neq\{1\}$ and so $\operatorname{Stab}_{G}\left(\beta\left(\alpha\left(p^{\prime}\right)\right)\right) \supseteq H_{1}$. Hence, $v\left(f\left(p^{\prime}\right)\right)=v(p) \in \operatorname{Sing}(X)$.

Corollary 4.7. Any rational curve in $X$ passes at least three times through singular points.

We will need the following consequence of Proposition 4.3.

COROLlARY 4.8. Let $S$ be a smooth surface of general type. Assume that $E$ is a $(-1)$-curve on $S$, then $E$ cannot intersect three distinct smooth rational curves with self-intersection -2 or -3 .

Proof. By Proposition 4.3, E intersects each of the three curves transversally in a point. Contracting $E$ we get three smooth rational curves with self-intersection -1 or -2 with a common point. If one of these has self-intersection -1 , by Corollary 4.4 , another curve has self-intersection -1 , and we find two intersecting $(-1)$-curves, which is impossible on a surface of general type. So all have self-intersection -2 .

We pass to the minimal model of $S$ by contracting all possible $(-1)$-curves. If one of the contracted curves intersected one of our three $(-2)$-curves, we get the same contradiction as above. So the image of our configuration gives three smooth rational curves with self-intersection -2 on a minimal surface of general type with a common point. This is impossible (see e.g. [13, Proposition 2]).

Proposition 4.3 and Corollary 4.8 imply that, if the basket of singularities of $X$ is simple enough, then $S$ is minimal. More precisely,

Proposition 4.9. Let $S \rightarrow X$ be a mixed q.e. surface of general type. Assume one of the following:

(i) Either all exceptional curves for $S \rightarrow X$ have self-intersection -2 or -3 ,

(ii) or $\mathcal{B}(X)=\left\{2 \times C_{4,1}, 3 \times C_{2,1}\right\}$.

Then $S$ is minimal.

Proof. (i) In this case, if $S$ were not minimal, then by Corollary 4.7 and Proposition 4.3 there would be a $(-1)$-curve $E$ which intersects three different smooth rational curves with self-intersection -2 or -3 , contradicting Corollary 4.8 . 
(ii) In this case the exceptional divisor is given by five rational curves which do not intersect each other, two of self-intersection -4 and three of self-intersection -2 . If $S$ were not minimal, then by Corollary 4.7, Proposition 4.3 and Corollary 4.4 the dual graph of the resulting configuration of rational curves would be:

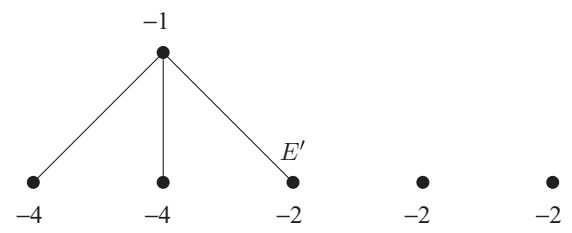

After the contraction of the $(-1)$-curve we can also contract $E^{\prime}$, finding a surface of general type with two $(-2)$ curves which are tangent in a point. Contracting all possible further $(-1)$-curves we find a contradiction as in the end of the proof of Corollary 4.8 .

Proposition 4.9 is obviously not sharp: We can prove the same result for many different baskets of singularities by exactly the same argument. We decided to state it in this weak form for the sake of simplicity, since a posteriori (inspecting the output of the program we describe in the next section) cases (i) and (ii) are the only cases that occur for mixed q.e. surfaces of general type with $p_{g}=0$ and $K^{2}>0$.

5. The classification. We wrote a MAGMA script which computes all mixed q.e. surfaces with fixed (input of the script) $p_{g}, q$ and $K^{2}$. To write the algorithm we needed to overcome some theoretical problems, namely to find explicit bounds for the basket of singularities $\mathcal{B}$ and for the signatures $\left(q ; m_{1}, \ldots, m_{r}\right)$.

For the basket of singularities, since by Corollary $2.19 B(\mathcal{B})=24\left(1-q+p_{g}\right)-$ $3 K^{2}$, it is enough to prove that there are finitely many possible baskets with fixed invariant $B(\mathcal{B})$, and show how to produce the whole list.

Lemma 5.1. Let $B_{0} \in \mathbb{Q}$. Then there are finitely many baskets $\mathcal{B}$ such that

$$
B(\mathcal{B})=B_{0}
$$

More precisely, $|\mathcal{B}| \leq B_{0} / 3$. Moreover, if $n / a=\left[b_{1}, \ldots, b_{l}\right]$ then

(1) $B\left(C_{n, a}\right) \geq \sum b_{i}$;

(2) $B\left(D_{n, a}\right) \geq 6+\frac{1}{2} \sum b_{i}$.

Proof. We note that $B\left(C_{n, a}\right)=\frac{a+a^{\prime}}{n}+\sum b_{i} \geq 3$, while $B\left(D_{n, a}\right)=\frac{B\left(C_{n, a}\right)}{2}+6 \geq 15 / 2>$ 3 : this proves $|\mathcal{B}| \leq B_{0} / 3$, bounding from above the number of singular points.

Claims (1) and (2) are trivial consequences of the definitions of $B\left(C_{n, a}\right)$ and $B\left(D_{n, a}\right)$; they show that there are only finitely many possible $\left[b_{1}, \ldots, b_{l}\right]$, so finitely many pairs $(n, a)$.

The second problem is to bound the possible signatures, once we have fixed $K^{2}$, $p_{g}, q$ and the basket $\mathcal{B}$. We have to find upper bounds for $r$ and $m_{i}$.

Definition 5.2. Let $\rho: S \rightarrow X=(C \times C) / G$ be a mixed q.e. surface. Let $\left(q ; m_{1}, \ldots, m_{r}\right)$ be the signature of the induced generating vector for $G^{0}$. Let $\mathcal{B}$ be 
the basket of singularities of $X$. Then we define the following numbers:

$$
\begin{gathered}
\Theta:=2 q(S)-2+\sum_{i=1}^{r}\left(\frac{m_{i}-1}{m_{i}}\right), \\
\beta:=\frac{12 \chi\left(\mathcal{O}_{S}\right)+k(\mathcal{B})-e(\mathcal{B})}{3 \Theta},
\end{gathered}
$$

Definition 5.3 (see [35]). The minimal positive integer $I_{x}$ such that $I_{x} K_{X}$ is Cartier in a neighbourhood of $x \in X$ is called the index of the singularity $x$. The index of a normal variety $X$ is the minimal positive integer $I$ such that $I K_{X}$ is Cartier. In particular, $I=\operatorname{lcm}_{x \in \operatorname{Sing}(X)} I_{x}$ depends only on the basket of singularities.

The index of a singularity of type $C_{n, a}$ is

$$
I_{x}=\frac{n}{\operatorname{gcd}(n, a+1)} .
$$

We can now give the bounds we need.

Proposition 5.4. Let $\rho: S \rightarrow X=(C \times C) / G$ be a mixed q.e. surface. Let $\left(q ; m_{1}, \ldots, m_{r}\right)$ be the signature of the induced generating vector for $G^{0}$. Let $\mathcal{B}=\mathcal{B}_{C} \cup \mathcal{B}_{D}$ be the basket of singularities of $X$. Then,

(a) $\Theta>0$ and $\beta=g(C)-1$;

(b) $r \leq 2 \Theta+4(1-q)$;

(c) $m_{i} \leq 4 \beta+6$;

(d) each $m_{i}$ divides $2 \beta I$, where $I$ is the index of $Y$;

(e) $m_{i} \leq \frac{2 I \beta \Theta+1}{M}$, with $M:=\max \left\{\frac{1}{6}, \frac{r-3+4 q}{2}\right\}$;

(f) except at most $\left|\mathcal{B}_{C}\right|+\left|\mathcal{B}_{D}\right| / 2$ indices $i, m_{i} \leq \frac{\beta \Theta+1}{M}$ and divides $\beta$.

Proof. (a) Since $q(S)=g\left(C / G^{0}\right)$, by the Hurwitz's formula:

$$
2(g(C)-1)=\left|G^{0}\right| \cdot \Theta,
$$

hence $\Theta=\frac{2(g(C)-1)}{\left|G^{0}\right|}>0$, since $g(C) \geq 2$. Let $k:=k(\mathcal{B})$ and $B:=B(\mathcal{B})$. By Corollary 2.19 and Proposition 2.18 we get

$$
\beta=\frac{24 \chi+3 k-B}{6 \Theta}=\frac{K_{S}^{2}+k}{2 \Theta}=\frac{8(g(C)-1)^{2}}{4 \Theta\left|G^{0}\right|}=g(C)-1 .
$$

(b) By definition $\Theta \geq 2 q-2+\frac{r}{2}$, hence $r \leq 2 \Theta-4(q-1)$.

(c) Since $m_{i}=\operatorname{ord}\left(h_{i}\right)$ and $h_{i}$ is an automorphism of a curve of genus $g \geq 2$, by Wiman's Theorem (see [40]) $m_{i} \leq 4 g+2=4 \beta+6$.

(d) Since $|\mathcal{B}(Y)|=2\left|\mathcal{B}_{C}\right|+\left|\mathcal{B}_{D}\right|$, the claim follows by [9, Proposition 1.14, d].

(e) We first show

$$
\Theta+\frac{1}{m_{i}} \geq \max \left\{\frac{1}{6}, \frac{r-3+4 q}{2}\right\} .
$$


Since $\Theta=2 q-2+r-\sum_{j=1}^{r} \frac{1}{m_{j}}$, we get

$$
\Theta+\frac{1}{m_{i}}=2 q-2+r-\sum_{j \neq i} \frac{1}{m_{j}} \geq 2 q-2+r-\frac{r-1}{2}=\frac{r-3+4 q}{2} .
$$

Since $\Theta>0, \frac{r-3+4 q}{2} \geq \frac{1}{6}$ unless $r=3$ and $q=0$. In this case, $\Theta>0$ implies that at most one $m_{i}$ can be equal to 2 . Hence, also in this case $\Theta+\frac{1}{m_{i}} \geq 0-2+3-\sum_{j \neq i} \frac{1}{m_{j}} \geq$ $1-\frac{1}{2}-\frac{1}{3}=\frac{1}{6}$. By $(\mathrm{d})$, we get

$$
\left(\max \left\{\frac{1}{6}, \frac{r-3+4 q}{2}\right\}\right) m_{i} \leq 1+\Theta \cdot m_{i} \leq 1+2 I \beta \Theta .
$$

(f) By [9, Proposition 1.14, e], except for at most $|\mathcal{B}(Y)| / 2=\left|\mathcal{B}_{C}\right|+\left|\mathcal{B}_{D}\right| / 2$ indices, $m_{i}$ divides $\beta$. From $m_{i} \leq \beta$, it follows that

$$
\left(\max \left\{\frac{1}{6}, \frac{r-3+4 q}{2}\right\}\right) m_{i} \leq 1+\Theta m_{i} \leq 1+\Theta \beta .
$$

We used the inequalities proved in this section to produce an algorithm to compute all mixed q.e. surfaces with fixed $p_{g}, q$ and $K^{2}$, following the same strategy of the algorithm of [9] which computed the product-quotient surfaces with $p_{g}=q=0$ (input was just $K^{2}$ ). The algorithm also uses the following simple remarks.

REMARK 5.5. By the Hurwitz's formula $|G|=2\left|G^{0}\right|=\frac{4(g(C)-1)}{\Theta}=\frac{4 \beta}{\Theta}$.

REMARK 5.6. Let $\rho: S \rightarrow X=(C \times C) / G$ be a mixed q.e. surface. Let $\left(q ; m_{1}, \ldots, m_{r}\right)$ be the signature of the induced generating vector for $G^{0}$. If $X$ has a singular point of type $C_{n, a}$ or $D_{n, a}$, then there exists $m_{i}$ such that $n$ divides $m_{i}$.

Indeed, the singular point is the class of a point $(x, y) \in C \times C$ such that $\operatorname{Stab}_{G^{0}}(x, y)=\langle\eta\rangle$ with $\operatorname{ord}(\eta)=n . x$ is a ramification point of $c: C \rightarrow C / G^{0}$, and its ramification index, which equals $\left|\operatorname{Stab}_{G^{0}}(x)\right|$, is one of the $m_{i}$. Since $\eta \in \operatorname{Stab}_{G^{0}}(x)$, it follows that $n$ divides $m_{i}$.

We explain here very briefly the strategy of the algorithm.

Having fixed the values of $K_{S}^{2}, p_{g}(S)$ and $q(S)$, by Corollary 2.19 we know $B(\mathcal{B})$, and Lemma 5.1 easily gives a procedure to produce the finite list of baskets with that invariant $B$. Then, for each basket, we produce the finite list of all signatures $\left(q ; m_{1}, \ldots, m_{r}\right)$ respecting all conditions in Proposition 5.4, including the requirement that $\beta$ is an integer.

Now, for each basket and for each associated signature, the orders of $G$ and $G^{0}$ are computed by Remark 5.5. Then the script checks all the finitely many groups $G^{0}$ of that order, and their unsplit degree 2 extensions $G$.

Then we have a list of quintuples (basket, signature, $G^{0}$, generating vector, extension), each quintuple gives a family of mixed q.e. surfaces (just determined by $\left(G^{0}\right.$, generating vector, extension) as explained in Remark 1.6), and all mixed q.e. surfaces 
Table 4. The skipped cases for $p_{g}=q=0$ and $K^{2}>0$

\begin{tabular}{lccc}
\hline$K_{S}^{2}$ & Sing $X$ & Sign. & $\left|G^{0}\right|$ \\
\hline 1 & $2 \times C_{8,1}, C_{4,1}$ & $2,3,8$ & 6,336 \\
1 & $3 \times C_{4,1}, C_{4,3}$ & $2,3,8$ & 2,304 \\
1 & $C_{8,1}, C_{4,1}, C_{8,5}$ & $2,3,8$ & 4,032 \\
1 & $4 \times C_{4,1}, C_{2,1}$ & $2,3,8$ & 2,880 \\
1 & $2 \times C_{8,3}, C_{4,1}, C_{2,1}$ & $2,3,8$ & 2,304 \\
1 & $2 \times C_{2,1}, C_{8,3}, C_{8,1}$ & $2,3,8$ & 3,744 \\
2 & $2 \times C_{8,3}, C_{4,1}$ & $2,3,8$ & 2,880 \\
2 & $C_{8,3}, C_{8,1}, C_{2,1}$ & $2,3,8$ & 4,320 \\
2 & $4 \times C_{4,1}$ & $2,4,5$ & 2,400 \\
2 & $4 \times C_{4,1}$ & $2,3,8$ & 3,456 \\
2 & $C_{8,3}, C_{8,5}, C_{2,1}$ & $2,3,8$ & 2,016 \\
2 & $2 \times C_{4,1}, 3 \times C_{2,1}$ & $2,3,8$ & 2,304 \\
2 & $2 \times C_{4,1}, C_{3,1}, C_{3,2}$ & $2,3,8$ & 2,496 \\
3 & $2 \times C_{4,1}, 2 \times C_{2,1}$ & $2,3,8$ & 2,880 \\
3 & $C_{8,3}, C_{8,1}$ & $2,3,8$ & 4,896 \\
3 & $2 \times C_{4,1}, C_{5,3}$ & $2,4,5$ & 2,160 \\
3 & $C_{8,3}, C_{8,5}$ & $2,3,8$ & 2,592 \\
3 & $C_{4,3}, C_{4,1}, C_{2,1}$ & $2,3,8$ & 2,304 \\
\hline
\end{tabular}

\begin{tabular}{lccc}
\hline$K_{S}^{2}$ & Sing $X$ & Sign. & $\left|G^{0}\right|$ \\
\hline 4 & $C_{4,3}, C_{4,1}$ & $2,3,8$ & 2,880 \\
4 & $4 \times C_{2,1}$ & $2,3,8$ & 2,304 \\
4 & $C_{3,1}, C_{3,2}, C_{2,1}$ & $2,3,8$ & 2,496 \\
4 & $2 \times C_{4,1}, C_{2,1}$ & $2,4,5$ & 2,400 \\
4 & $2 \times C_{4,1}, C_{2,1}$ & $2,3,8$ & 3,456 \\
5 & $C_{5,2}, C_{2,1}$ & $2,4,5$ & 2,160 \\
5 & $3 \times C_{2,1}$ & $2,3,8$ & 2,880 \\
5 & $C_{3,1}, C_{3,2}$ & $2,3,8$ & 3,072 \\
5 & $2 \times C_{4,1}$ & $2,4,5$ & 2,800 \\
5 & $2 \times C_{4,1}$ & $2,3,8$ & 4,032 \\
& $2 \times C_{2,1}$ & $2,4,5$ & 2,400 \\
6 & $2 \times C_{2,1}$ & $2,3,8$ & 3,456 \\
6 & $2 \times C_{5,3}$ & $2,4,5$ & 2,560 \\
6 & $C_{2,1}$ & $2,3,9$ & 2,268 \\
7 & $C_{2,1}$ & $2,4,5$ & 2,800 \\
7 & $C_{2,1}$ & $2,3,8$ & 4,032 \\
7 & $\emptyset$ & $2,3,9$ & 2,592 \\
8 & $\emptyset$ & $2,4,5$ & 3,200 \\
8 & $\emptyset$ & $2,3,8$ & 4,608 \\
8 & & &
\end{tabular}

with the prescribed invariants are here. Anyway, in this list there are also surfaces with different invariants: those whose singularities do not correspond with the basket. Then the script computes the singularities in each case, using the results of Section 2 (in particular, Propositions 2.1 and 2.3), and it discards the surfaces with wrong basket.

Moreover, different generating vectors give isomorphic surfaces if they differ by some Hurwitz moves, which are described, in the cases we need, in [29, Section 5]. The script computes this action on the remaining generating vectors, and returns only a representative for each orbit. Finally, the script computes, using a result by Armstrong $[\mathbf{1}, \mathbf{2}]$, the fundamental groups (see [24]) of the resulting surfaces.

Our code skips some signatures giving rise to groups of large order, either not covered by the MAGMA SmallGroup database or causing extreme computational complexity. The program returns the list of skipped cases, which have to be studied separately.

A commented version of the full program can be downloaded from: http: / / www. science. unitn. it/ pignatel/papers/Mixed. magma

Using it, we proved Theorems A, B and $\mathrm{C}$ as follows.

Sketch of the proof of Theorems $A, B$ and $C$. By Corollary 2.19, every mixed q.e. surface has $K^{2} \leq 8 \chi$; so the possible invariants of a minimal surface of general type with $\chi=1$ are $K_{S}^{2}=1,2,3,4,5,6,7,8$ and, by Beauville's inequality [12], $p_{g} \geq 2 q-4$, $p_{g}=q=\{0,1,2,3,4\}$. We ran our program for all these values; it returned the surfaces in Tables 1, 2 and 3 .

As mentioned, the surfaces returned by the program may not be all mixed q.e. surfaces with the required invariants, since the program is forced to skip some signatures, giving rise to groups of large order. The program returns the list of these 'skipped' cases.

For the cases $p_{g}=q \neq 0$, this list is empty, so Tables 2 and 3 are complete. We report the list of 'skipped' signatures for $p_{g}=q=0$ in Table 4 . We proved that none of these 
cases occur by arguments very similar to the analogous proofs in the papers $[\mathbf{6}, \mathbf{9}, \mathbf{2 4}]$ and therefore we do not include these here. Interested readers will find the details at http: / / www. science. unitn. it/ pignatel/papers/skipped.pdf

Now let us consider the surfaces given in Tables 1, 2 and 3. A surface with $K^{2}>0$ is either of general type or rational, therefore regular and simply connected: a quick inspection of the tables shows that this latter case does not occur, so all constructed surfaces are of general type. By Theorem 4.5 and Proposition 4.9, all the constructed surfaces are minimal. Moreover, again by Proposition 4.5, since every minimal surface of general type has positive $K^{2}$, we have found all irregular mixed q.e. surfaces with $p_{g}=q$.

ACKnOwledgements. The authors are indebted to M. Penegini for his careful reading of the first version of this manuscript; in particular the current forms of Proposition 2.21 and Corollary 2.20 are due to him, authors had proven only a weaker inequality. The authors are grateful to I. Bauer and F. Catanese for several inspiring conversations on group actions on the products of two curves. Some of the results in this paper were developed in Fall 2012, when the first author was supported by the Department of Mathematics of the University of Trento. The second author was partially supported by the FCT Project PTDC/MAT/111332/2009 Moduli Spaces in Algebraic Geometry, the PRIN 2010-2011 Project Geometria delle varietà algebriche and the FIRB 2012 Project Spazi di moduli e applicazioni. Both authors are members of G.N.S.A.G.A. of I.N.d.A.M.

\section{REFERENCES}

1. M. A. Armstrong, On the fundamental group of an orbit space, Proc. Camb. Phil. Soc. 61 (1965), 639-646.

2. M. A. Armstrong, The fundamental group of the orbit space of a discontinuous group, Proc. Camb. Phil. Soc. 64 (1968), 299-301.

3. A. P. Barth, K. Hulek, C. A. M. Peters and A. Van de Ven, Compact complex surfaces, vol. 4 (Springer, Berlin, Germany, 2004) (Ergebnisse der Mathematik und ihrer Grenzgebiete).

4. I. Bauer and F. Catanese, Some new surfaces with $p_{g}=q=0$, in The Fano Conference (Alberto C., Alberto C. and Marina M., Editors) (Universita di Torino, Dipartimento di Matematica, Turin, 2004), 123-142.

5. I. Bauer, F. Catanese and F. Grunewald, The classification of surfaces with $p_{g}=q=0$ isogenous to a product of curves, Pure Appl. Math. Q. 4(2) (2008), 547-586.

6. I. Bauer, F. Catanese, F. Grunewald and R. Pignatelli, Quotients of products of curves, new surfaces with $p_{g}=0$ and their fundamental groups, Am. J. Math. 134(4) (2012), 993-1049.

7. I. Bauer, F. Catanese and R. Pignatelli, Complex surfaces of general type: Some recent progress, in Global aspects of complex geometry (Springer, Berlin, Germany, 2006), pp. 1-58.

8. I. Bauer, F. Catanese and R. Pignatelli, Surfaces of general type with geometric genus zero: A survey, in Complex and differential geometry, vol. 8, Springer Proceedings in Mathematics (Springer, Berlin, Germany, 2011), pp. 1-48.

9. I. Bauer and R. Pignatelli, The classification of minimal product-quotient surfaces with $p_{g}=0$, Math. Comput. 81(280) (2012), 2389-2418.

10. I. Bauer and R. Pignatelli, Product-quotient surfaces: New invariants and algorithms. arXiv:1308.5508 [math.AG] (preprint 2013). 1978)

11. A. Beauville, Surfaces algébriques complexes (Société Mathématique de France, Paris,

12. A. Beauville, L' inégalité $p_{g} \geq 2 q-4$ pour les surfaces de type général, Bull. Soc. Math. France 110(3) (1982), 343-346.

13. E. Bombieri, Canonical models of surfaces of general type, Inst. Hautes Études Sci. Publ. Math. 42 (1973), 171-219. 

358.

14. E. Brieskorn, Rationale singularitäten komplexer Flächen, Invent. Math. 4 (1968), 336

15. G. Carnovale and F. Polizzi, The classification of surfaces with $p_{g}=q=1$ isogenous to a product of curves, $A d v$. Geom. 9(2) (2009), 233-256.

16. F. Catanese, Fibred surfaces, varieties isogenous to a product and related moduli spaces, Am. J. Math. 122(1) (2000), 1-44.

17. F. Catanese, Q.E.D. for algebraic varieties, J. Differ. Geom. 77(1) (2007), 43-75.

18. F. Catanese, On a class of surfaces of general type, in Algebraic surfaces, C.I.M.E. Summer Sch., vol. 76 (Springer, Heidelberg, Germany, 2010), pp. 267-284.

19. F. Catanese and C. Ciliberto, Surfaces with $p_{g}=q=1$, in Problems in the theory of surfaces and their classification (Cortona, 1988) (Catanese F. et al., Editors), Sympos. Math., XXXII (Academic Press, London, 1991), pp. 49-79.

20. F. Catanese and C. Ciliberto, Symmetric products of elliptic curves and surfaces of general type with $p_{g}=q=1$. J. Algebr. Geom. 2(3) (1993), 389-411.

21. F. Catanese, C. Ciliberto and M Mendes Lopes, On the classification of irregular surfaces of general type with nonbirational bicanonical map, Trans. Amer. Math. Soc. 350(1) (1998), 275 308 .

22. F. Catanese and R. Pignatelli, Fibrations of low genus. I, Ann. Sci. École Norm. Sup. (4) 39(6) (2006), 1011-1049.

23. F. Cossec and I. Dolgachev, Enriques Surfaces I (Birkhäuser, Heidelberg, Germany, 1989).

24. D. Frapporti, Mixed surfaces, new surfaces of general type with $p_{g}=0$ and their fundamental group, Collect. Math. 64(3) (2013), 293-311.

25. C. D. Hacon and R. Pardini, Surfaces with $p_{g}=q=3$, Trans. Amer. Math. Soc. 354(7), (2002), 2631-2638 (electronics).

26. W. Bosma, J. Cannon and C. Playoust, The Magma algebra system. I. The user language, J. Symbolic Comput 24(3-4) (1997), 235-265.

27. K. Matsuki, Introduction to the Mori program, Graduate Texts in Mathematics, vol. 56 (Springer-Verlag, Heidelberg, Germany, 2002).

28. E. Mistretta and F. Polizzi, Standard isotrivial fibrations with $p_{g}=q=1$, II, J. Pure Appl. Algebr. 214(4) (2010), 344-369.

29. M. Penegini, The classification of isotrivially fibred surfaces with $p_{g}=q=2$, Collect. Math. 62(3) (2011), 239-274. (With an appendix by Sönke Rollenske.)

30. R. Pignatelli, Some (big) irreducible components of the moduli space of minimal surfaces of general type with $p_{g}=q=1$ and $K^{2}=4$, Atti. Accad. Naz. Lincei Cl. Sci. Fis. Mat. Natur. Rend. Lincei (9) Mat. Appl. 20(3) (2009), 207-226.

31. G. P. Pirola, Surfaces with $p_{g}=q=3$, Manuscr. Math. 108(2) (2002), 163-170.

32. F. Polizzi, On surfaces of general type with $p_{g}=q=1$ isogenous to a product of curves, Comm. Algebra 36(6) (2008), 2023-2053. 1631.

33. F. Polizzi, Standard isotrivial fibrations with $p_{g}=q=1$, J. Algebra 321(6) (2009), 1600

34. F. Polizzi, Numerical properties of isotrivial fibrations, Geom. Dedicata 147 (2010), 323-355.

35. M. Reid, Young person's guide to canonical singularities, in Algebraic geometry Bowdoin 1985, Part 1 (Brunswick, Maine, 1985), Proc. Sympos. Pure Math., vol. 46 (American Mathematical Society, Providence, RI, 1987), pp. 345-414.

36. C. Rito, On surfaces with $p_{g}=q=1$ and non-ruled bicanonial involution, Ann. Sc. Norm. Super. Pisa Cl. Sci. (5) 6(1) (2007), 81-102.

37. C. Rito, Involutions on surfaces with $p_{g}=q=1$, Collect. Math. 61(1) (2010), 81-106.

38. C. Rito, On equations of double planes with $p_{g}=q=1$, Math. Comp. 79(270) (2010), $1091-1108$.

39. F. Serrano, Isotrivial fibred surfaces, Ann. Mat. Pura Appl. 171(4) (1996), 63-81.

40. A. Wiman, Über die hyperelliptischen Kurven und diejenigen vom Geschlechte $p=$ 3, welche eindeutige transformationen in sich zulassen, Bihang Kongl. Svenska VetenkampsAkademiens Handlingar 21 (1895), 1-23.

41. F. Zucconi, Surfaces with $p_{g}=q=2$ and an irrational pencil, Canad. J. Math. 55(3) (2003), 649-672. 